\title{
REACTION TIMES TO \\ HIGH-CONTRAST BRAKE LAMPS
}

Michael Sivak

Michael Flannagan

Andrew W. Gellatly

The University of Michigan

Transportation Research Institute

Ann Arbor, Michigan 48109-2150

U.S.A.

Report No. UMTRI-90-30

July 1990 
Technical Report Documentation Page

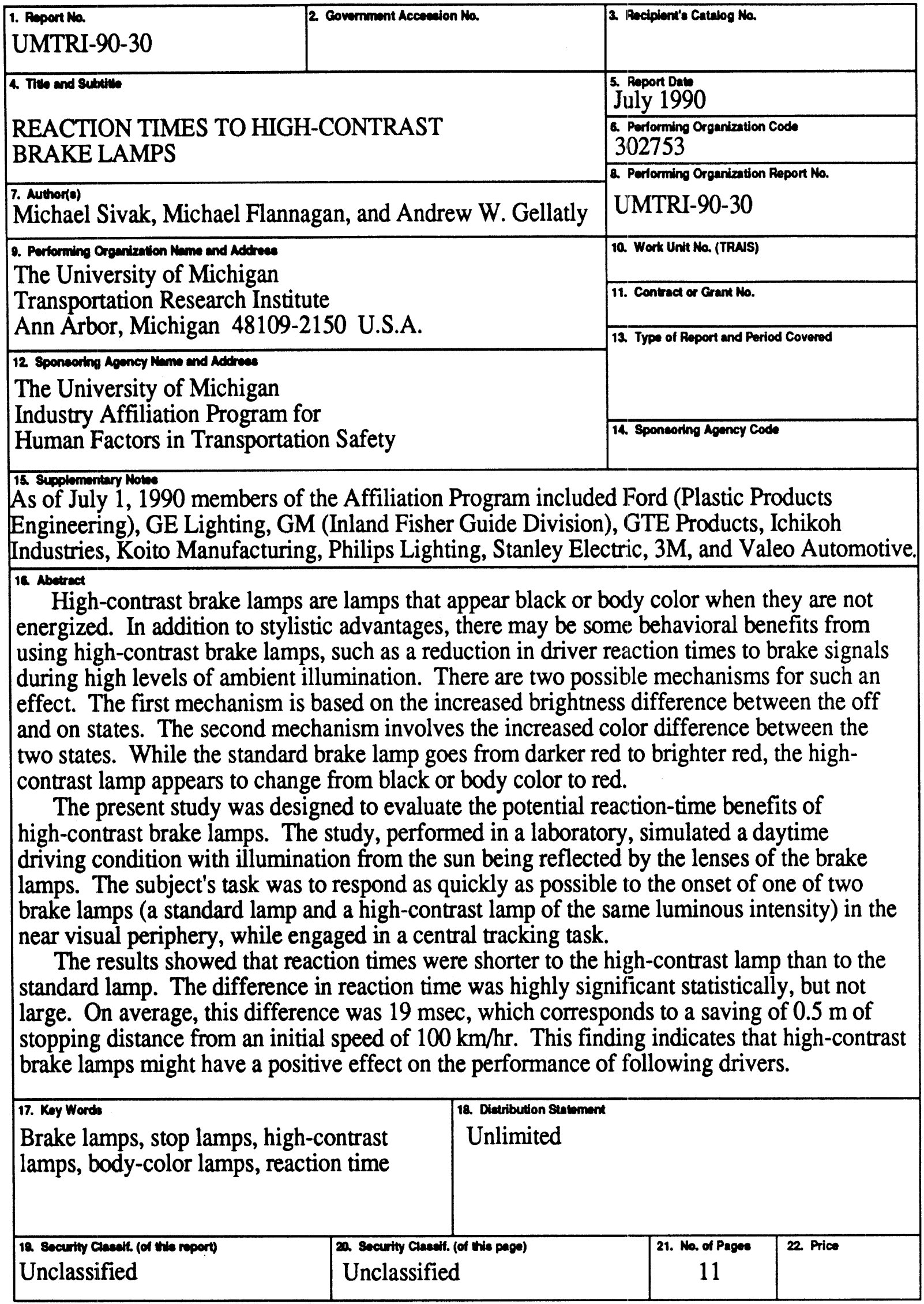




\title{
ACKNOWLEDGEMENT
}

Appreciation is extended to the current members of The University of Michigan Industry Affiliation Program for Human Factors in Transportation Safety for support of this research:

\author{
Ford (Plastic Products Engineering) \\ GE Lighting \\ GM (Inland Fisher Guide Division) \\ GTE Products \\ Ichikoh Industries \\ Koito Manufacturing \\ Philips Lighting \\ Stanley Electric \\ $3 \mathrm{M}$ \\ Valeo Automotive
}




\section{CONTENTS}

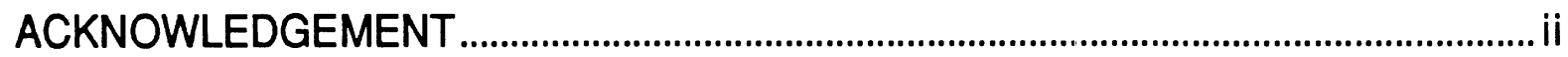

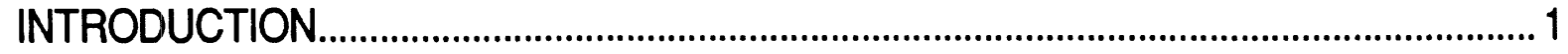

METHOD

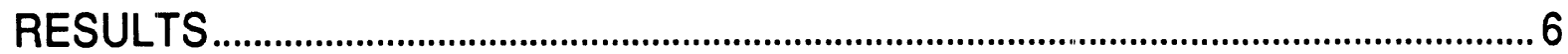

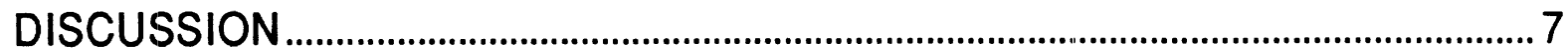




\section{INTRODUCTION}

The dominant perceptual change when traditional brake lamps go from the "off" to the "on" state is the change in the apparent brightness. In the off state the apparent brightness is determined primarily by the amount of ambient light reflected by the brake-lamp lens towards the eyes of the observer, and light passing into and out of the lamp. In the on state, the light emitted by the bulb is added to the reflected light, leading to an increase in perceived brightness. At low levels of ambient illumination, the difference between the light reaching the observer in the off and on stages is substantial, because the amount of reflected light is relatively negligible in relation to the emitted light. However, that might not be the case at high levels of ambient illumination, especially if there is significant reflection from the sun. Under these conditions the perceptual change from the off to the on state might be small enough to cause confusion about the actual state of the lamps and, consequently, lead to increased reaction time to the onset of the brake signals.

In recent years, spurred originally by stylistic considerations, some manufacturers have employed brake lamps that, in the off state, appear black or the same color as the surrounding car body. This effect is achieved by covering the lens of the lamps by narrow black or body-color stripes.

In addition to stylistic advantages, there may be some behavioral benefits from using high-contrast brake lamps, such as a reduction in driver reaction times to brake signals during high levels of ambient illumination. There are two possible mechanisms for such an effect. The first mechanism is based on the increased brightness difference between the off and on states. The second mechanism involves the increased color difference between the two states. While the standard brake lamp goes from darker red to brighter red, the high-contrast lamp appears to change from black or body color to red.

The present study was designed to evaluate the potential reaction-time benefits of high-contrast brake lamps. The study, performed in a laboratory, simulated a daytime driving condition with illumination from the sun being reflected by the lenses of the brake lamps. 


\section{METHOD}

\section{Tasks}

The subjects were asked to perform two concurrent tasks. The primary task was to respond as quickly as possible to the onset of one of two brake lamps in the near visual periphery. The secondary task, which was designed to approximate the perceptual and motor workload of driving, involved continuous compensatory tracking. This task was presented on a television monitor. Deviations of the road's center were based upon a sinusoidal sequence which repeated about every minute. However, since the subject was kept busy with two tasks, to the subject the road appeared to be curving in an unpredictable manner. The subject's task was to keep the road centered on the screen by use of a knob.

\section{Equipment}

Schematic diagrams of the experimental set-up and the subject's view are shown in Figures 1 and 2. The subject sat at one end of a table. Directly in front of the subject, at a distance of $4 \mathrm{~m}$, there was a black board on another table. This board, positioned perpendicularly to the subject's line of sight, was $152.9 \mathrm{~cm}$ wide and 50.8 $\mathrm{cm}$ high. There were three apertures in the board. One aperture, $48.3 \mathrm{~cm}$ wide and $38.8 \mathrm{~cm}$ high, revealed a television monitor which was positioned immediately behind the board. The other two apertures, each $11.1 \mathrm{~cm}$ wide and $7.6 \mathrm{~cm}$ high, revealed a pair of brake lamps, also positioned immediately behind the board. From the subject's point of view, the visual angle between the center of the monitor and the center of either the left or the right brake lamp was $7.9^{\circ}$.

Photoflood lamps were used to illuminate the face of each of the brake lamps at a level of 36,544 Ix, to simulate illumination from the sun. The room was also lighted with ceiling-mounted fluorescent lamps. The resulting non-uniform background luminance was measured at two locations. On the black board, immediately above the monitor, the luminance was $11.1 \mathrm{~cd} / \mathrm{m}^{2}$, while on the grey wall immediately above the center of the board the luminance was $71.2 \mathrm{~cd} / \mathrm{m}^{2}$.

A microcomputer randomized inter-stimulus intervals and selection of the lamp to be energized, and recorded subject's reaction times. 

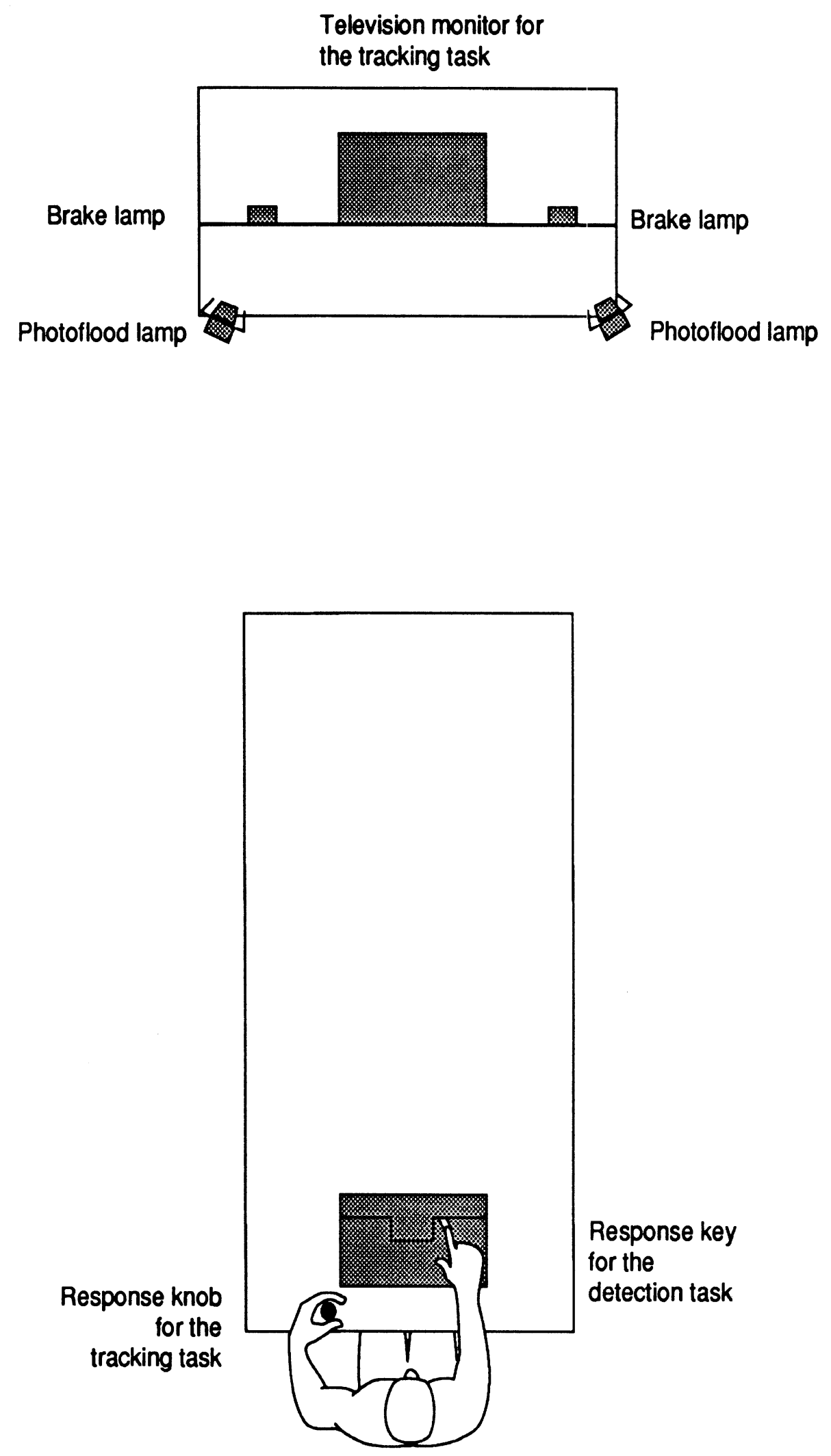

Figure 1. A schematic diagram of the experimental setup. 


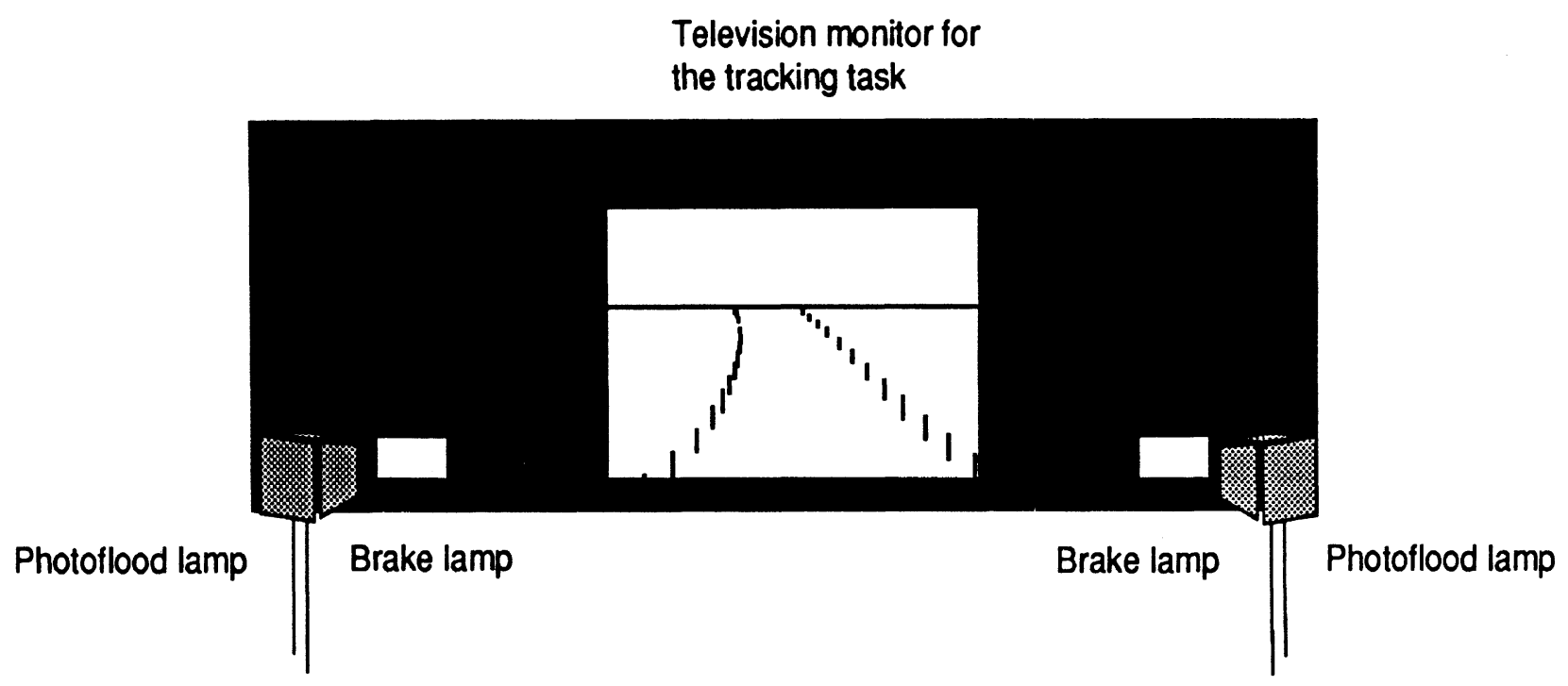

Figure 2. A schematic diagram of the subject's view.

\section{Brake lamps}

Two brake lamps were tested. One was a standard brake lamp. The other lamp, to be referred to here as the high-contrast brake larnp, was a modified standard lamp. The modification involved placing thin horizontal stripes of black material across the lens. These stripes were $3 \mathrm{~mm}$ wide, placed $2 \mathrm{~mm}$ apart. The stripes were placed at the junctions between the lens pillows. Additionally, to help equalize emitted light, the light output of the standard brake lamp was reduced by use of a clear plastic sheeting with a transmissivity of $79 \%$. Both lamps used the same type of bulb-No. 2057.

The illuminance at the subject's eye point was the same for both of the brake lamps--6.1 Ix. Treating the lamps as point sources, the calculated luminous intensity directed towards the eyes of the subject was $100.4 \mathrm{~cd}$ for each lamp. The $x, y$ chromaticities of the two lamps were $.69, .30$ (standard off), $.69, .31$ (standard on), .46 , .38 (high contrast off), and .68, .32 (high contrast on). 


\section{Subjects}

Twenty paid subjects participated in this study. There were five subjects in each of the following groups: younger males, younger females, older males, and older females. (One older male was replaced because of his difficulties with the tracking task.) The ages of the younger subjects ranged from 20 to 23 years with a mean of 21.4 , and the ages of older subjects ranged from 61 to 78 with a mean of 67.7 .

\section{Photometry checks}

To insure that photometric levels remained stable over the period in which data were collected, the luminous intensity of the two brake lamps (in both positions) and illumination from the photoflood lamps were spot checked throughout the study. The average coefficient of variation (standard deviation divided by the mean) was 0.3 percent for measurements of both the brake lamps and the photoflood lamps.

\section{Procedure}

Subjects were tested individually. Each subject was first given several minutes of practice on the tracking task using the left hand, until he or she felt comfortable with it. At this point the reaction-time task was introduced and explained. On each trial either the left or the right brake lamp was energized for $2 \mathrm{sec}$. The subject was instructed to respond as quickly as possible to the onset of either lamp by depressing a response key with the right forefinger. The inter-stimulus interval (time separation between two successive presentations of the brake lamps) was either 10, 15, 20, 25, or $30 \mathrm{sec}$, with each value presented an equal number of times. The subject was asked to continue the tracking task throughout the entire block of trials, keeping the eyes focused approximately at the center of the television monitor.

There were 10 trial types ( 2 brake lamps $\times 5$ inter-stimulus intervals). The study consisted of a practice block of 10 trials (a randomized ordering of each of the trial types), and two experimental block of 40 trials each (a randomized ordering of four replications of each trial type per block). The locations of the two brake lamps were exchanged during the break between the two experimental blocks. For one half of the subjects the standard brake lamp was on their right during the practice block and the first experimental block; for the other half the standard brake lamp was initially on their left.

A short break was given between the two experimental blocks of trials. The complete test session took about 30 minutes per subject. 


\section{RESULTS}

The reaction times were submitted to an analysis of variance using the following factors: lamp type (standard or high contrast), age group (younger or older), and sex. The effect of lamp type was statistically significant, $F(1,16)=22.05, p<$ .0002 , with faster reaction times for the high-contrast lamp than for the standard lamp (439 vs. $458 \mathrm{msec}$ ). The effect of age group was also significant, $F(1,16)=22.60, p<$ .0002 , with younger subjects responding faster than older subjects (404 vs. 493 msec). The effect of sex was marginally not significant, $F(1,16)=4.01, p=.06$, with males tending to respond faster than females ( $429 \mathrm{vs} .467 \mathrm{msec}$ ). None of the interactions was significant. Figure 3 is a graphic representation of one of these nonsignificant interactions (lamp type and age group): the reaction-time difference between standard and high-contrast lamps was the same $(19 \mathrm{msec})$ for both younger and older subjects.

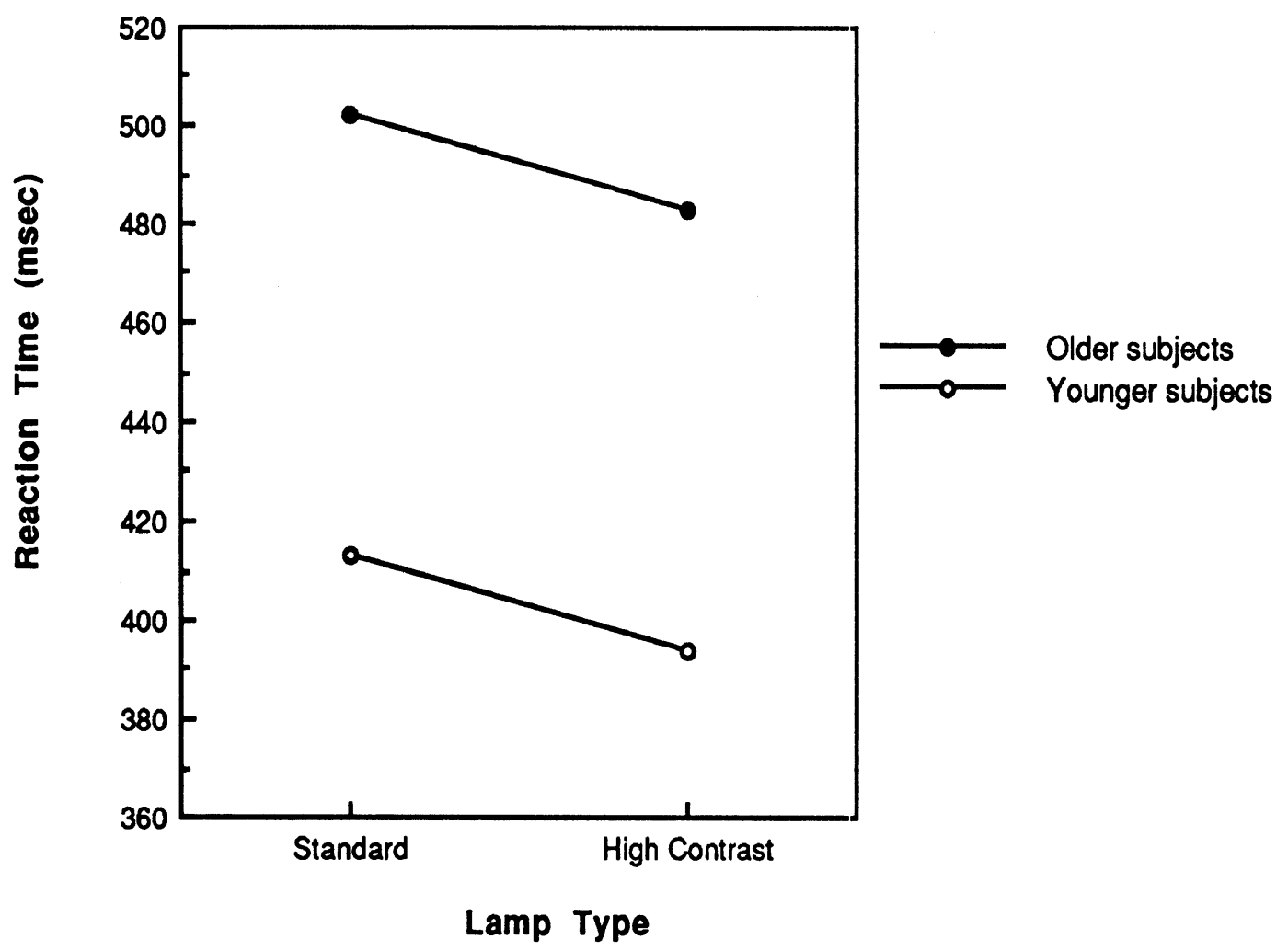

Figure 3. Reaction time as a function of lamp type and age group. 


\section{DISCUSSION}

This study evaluated the potential benefits of high-contrast brake lamps--lamps that appear black or body-color when they are not energized. Specifically, this study compared reaction times to brake signals from standard and high-contrast brake lamps of the same luminous intensity. Reaction times to the high-contrast lamp were shorter than reaction times to the standard lamp. The difference in reaction time was highly significant statistically, but not large. On average, this difference was $19 \mathrm{msec}$, which corresponds to a saving of $0.5 \mathrm{~m}$ of stopping distance from an initial speed of $100 \mathrm{~km} / \mathrm{hr}$.

The present data do not provide complete information about the mechanisms responsible for the difference in reaction times to the two lamps. Nevertheless, some inferences are possible. First, the only salient differences between the two lamps were in how much they changed brightness or chromaticity between the off and on states. It is therefore reasonable to assign the reaction-time effect to relative brightness change, relative chromaticity change, or to both. However, these results do not indicate which of those factors is more important in determining reaction time. Second, it is interesting that the effect of the high-contrast lamp is the same for younger and older subjects (as shown by the lack of interaction in Figure 3). This is exactly the pattern of results that one would expect if the high-contrast lamp simply came on $19 \mathrm{msec}$ earlier than the standard lamp, relative to their nominal onset times. Different groups of subjects might differ in overall reaction time (as the younger and older subjects do), but the effect of lamp type would have to be the same for both groups because the basis for the effect would be in the stimulus itself rather than in subjects' responses to it. Alternatively, suppose that the cause of the reaction time difference involved more elaborate aspects of how subjects responded to it, as might be the case if one lamp were more salient or compeling. Because of the overall difference in reaction time between younger and older subjects, we know that there must be differences between those groups in the various cognitive processes that take place between a stimulus and the response to it. If lamp type affected those processes, we might expect that in addition to the difference between younger and older subjects in overall reaction times (the main effect of age), there would also be a difference between older and younger subjects in the difference in reaction time due to lamp type (an interaction effect of age and lamp type). The lack of such an interaction therefore makes it a better bet that the effect of lamp type is due to early sensory mechanism or even stimulus mechanism. That is consistent with the 
possibility that the higher off-state luminance of the standard lamp masks its onset for 19 msec relative to the onset of the high-contrast lamp.

The obtained reaction-time saving applies to daytime situations with high levels of ambient illumination impinging on the faces of the lamps. Such situations present the most difficult conditions for detecting brake signals. As the ambient illumination is decreased, the reaction-time savings should be reduced. Furthermore, the effect is likely to be absent altogether for nighttime situations with the presence lamps energized. In such situations both the brightness and chromaticity differences between the off and on states would be approximately the same for both the normal and high-contrast lamps (assuming the traditional U.S. system of presence and brake signals presented in the same lamp cavity).

In the present study, the brake signals came from one of two spatial locations that were known to the subject. In real life, a high-contrast brake lamp (in the off state) might perceptually blend with the rest of the car body. Such situation might lead to a certain degree of spatial uncertainty as to where brake signals might appear, although the uncertainty region would still be limited to the rear of the vehicle. Whether this somewhat increased location uncertainty would lead to an increase in reaction times is yet unknown.

The original motivation for the use of high-contrast brake lamps was based on aesthetic considerations. The present findings indicate not only that such lamps might have no detrimental effects on the performance of following drivers, but also that they might have a positive effect. To fully confirm this conjecture, the potential problems with the increased spatial uncertainty would have to be experimentally ruled out. 